\title{
ESTUdO COMPARATIVO dOS PROCEDIMENTOS PERCUTÂNEOS ORIENTADOS POR MÉTODOS DE IMAGEM NO TRATAMENTO DAS COLEC̣̃̃ES INTRAPERITONEAIS*
}

\author{
Giselle Guedes Netto de Mello ${ }^{1}$, Gustavo de Souza Portes Meirelles ${ }^{2}$, José Marcelo Amatuzzi \\ de Oliveira ${ }^{1}$, Sergio Ajzen ${ }^{3}$, Giuseppe D'Ippolito ${ }^{4}$, Jacob Szejnfeld ${ }^{5}$
}

Resumo OBJETIVO: Verificar e comparar a eficácia da aspiração percutânea (AP) e da drenagem percutânea (DP), orientadas por métodos de imagem, no tratamento das coleções intraperitoneais. MATERIAIS E MÉTODOS: Foram encaminhados ao nosso setor 52 pacientes para tratamento percutâneo de coleções abdominais. Destes, 16 apresentavam coleções intraperitoneais, sendo dez complexas (multiloculadas ou múltiplas) e seis, simples. $O$ volume das coleções variou de 40 a $1.200 \mathrm{ml}$, com mediana de $200 \mathrm{ml}$. Foram submetidas a AP seis coleções $(37,5 \%)$ e a DP, dez $(62,5 \%)$. Considerou-se como sucesso do método a resolução completa da coleção, com melhora clínico-laboratorial do paciente. RESULTADOS: 0 sucesso do procedimento ocorreu em $75 \%$ dos casos. Nas coleções submetidas a AP houve sucesso em $100 \%$, e nas submetidas a DP o sucesso foi de $60 \%$. 0 índice de sucesso no grupo submetido a DP foi de $50 \%$ nas coleções menores ou iguais a $200 \mathrm{ml}$ e de $75 \%$ nas de maior volume. Obteve-se sucesso com a DP em 33,3\% das coleções complexas, em $42,9 \%$ das infectadas e em $100 \%$ das não infectadas e simples. Foram observadas apenas duas complicações menores (bacteremia transitória e infecção cutânea) após os procedimentos. CONCLUSÃO: A AP e a DP são métodos eficazes e seguros para tratamento das coleções intraperitoneais. No nosso estudo, a AP foi mais eficaz que a DP, principalmente nas coleções de menor volume. Unitermos: Coleções abdominais; Aspiração percutânea; Drenagem percutânea.

Abstract A comparison study between image-guided percutaneous procedures in the treatment of intra-abdominal liquid collections.

OBJECTIVE: To determine and compare the efficacy of image-guided percutaneous needle aspiration (PNA) versus percutaneous catheter drainage (PCD) in the treatment of intra-abdominal liquid collections. MATERIALS AND METHODS: A prospective study involving 52 patients referred to our service for percutaneous treatment of abdominal liquid collections was carried out. Sixteen of these patients had intra-abdominal liquid collections (six patients with simple localized collections and ten patients with complex, multiseptated or multiple, collections). The volume of the collections ranged from 40 to 1,200 $\mathrm{ml}$ (median, $200 \mathrm{ml}$ ). PCD was performed in ten $(62.5 \%)$ collections and PNA in six $(37.5 \%)$ collections. Successful treatment was defined as complete resolution of the collection with both clinical and laboratorial recovery. RESULTS: Success was achieved in $75 \%$ of cases. The success rate was $100 \%$ and $60 \%$ in the groups submitted to PNA and PCD, respectively. Successful treatment was observed in $50 \%$ of the patients submitted to PCD with collections $\leq \mathbf{2 0 0} \mathrm{ml}$, and in $\mathbf{7 5} \%$ of the patients with larger collections. Complete resolution with PCD was obtained in $33.3 \%$ of complex, $42.9 \%$ of infected and $100 \%$ of simple or sterile collections. Only two minor complications (transient bacteremia and cutaneous infection) due to the procedures were observed. CONCLUSION: Our results showed that PCD and PNA are safe and effective in the treatment of intra-abdominal collections. In this study, needle aspiration was more effective than drainage, particularly for smaller collections. Key words: Intra-abdominal collections; Percutaneous needle aspiration; Percutaneous catheter drainage.

\footnotetext{
* Trabalho realizado no Departamento de Diagnóstico po Imagem da Universidade Federal de São Paulo/Escola Paulista de Medicina (Unifesp/EPM), São Paulo, SP.

1. Médicos Doutores em Radiologia Clínica pela Unifesp/EPM

2. Pós-graduando do Departamento de Diagnóstico por Imagem da Unifesp/EPM.

3. Professor Adjunto Livre-Docente do Departamento de Diagnóstico por Imagem da Unifesp/EPM.

4. Professor Visitante do Departamento de Diagnóstico por Imagem da Unifesp/EPM.

5. Professor Livre-Docente, Chefe do Departamento de Diagnóstico por Imagem da Unifesp/EPM.

Endereço para correspondência: Dra. Giselle Guedes Netto de Mello. Rua Antônio Lucas de Oliveira, 27, Veleiros. São Paulo, SP, 04773-060. E-mail: giselle.mello@fleury.com.br

Recebido para publicação em $21 / 5 / 2003$. Aceito, após revisão, em 1/7/2003.
}

\section{INTRODUÇÃO}

Apesar do grande progresso das técnicas cirúrgicas e da disponibilidade de potentes antibióticos, o tratamento das coleções intraperitoneais, especialmente das infectadas, continua delicado ${ }^{(1,2)}$.

Essas coleções constituem afecções graves, geralmente decorrentes de procedimentos cirúrgicos ou de processos inflamatórios como apendicite, diverticulite ou pancreatite ${ }^{(3,4)}$. Cursam com altos índices de mortalidade, em torno de $80 \%$ a $100 \%$ se não adequadamente tratadas ${ }^{(5-7)}$.

O tratamento eficaz depende da drenagem precoce dessas coleções, associada a antibioticoterapia específica ${ }^{(\mathbf{3 , 4 , 8 , 9})}$. O método terapêutico tradicionalmente utilizado corresponde ao esvaziamento cirúrgico, que exige anestesia geral e associa-se a índices significativos de morbidade e mortalidade $^{(2,3,10-13)}$. 
Nos últimos anos, com o desenvolvimento de novos métodos radiológicos diagnósticos e terapêuticos, uma nova abordagem das coleções intraperitoneais tem sido possível. A avaliação radiológica por técnicas seccionais, como a ultra-sonografia (US), a tomografia computadorizada (TC) e a ressonância magnética (RM), permite maior sensibilidade e especificidade na detecção e caracterização da coleção, em comparação aos métodos radiológicos convencionais e às técnicas cirúrgicas $^{(\mathbf{3}, \mathbf{1 2}, \mathbf{1 4 , 1 5})}$. Além disso, estes métodos permitem orientação segura para procedimentos menos invasivos, como a aspiração percutânea (AP) e a drenagem percutânea (DP), que se tornaram alternativas satisfatórias à cirurgia convencional, com taxas semelhantes ou superiores de sucesso, porém com custo inferior, redução no tempo de internação hospitalar e menores índices de morbimortalidade $\mathrm{e}^{\mathbf{4 , 1 1 - 1 4})}$.

Entre os métodos disponíveis para o tratamento percutâneo das coleções intraperitoneais, a DP é técnica consagrada, sendo para muitos autores a primeira escolha, por permitir manutenção de drenos, com controle preciso do esvaziamento da coleção $^{(\mathbf{2 , 8 - 1 1 , 1 3 , 1 6 )}}$. A AP, apesar de constituir método simples, rápido, barato e seguro para o paciente, tem sido utilizada apenas como procedimento diagnóstico ${ }^{(\mathbf{1 7 - 1 9 )}}$, apesar da opinião de alguns autores, que ressaltam uma eficiência equivalente e cus-

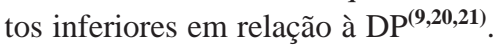

\section{Objetivo}

Como poucos estudos comparam os resultados obtidos no tratamento percutâneo das coleções intraperitoneais pela AP e DP, realizamos análise prospectiva com o objetivo de verificar e comparar a eficácia destes procedimentos, orientados por métodos de imagem, no tratamento dessas coleções.

\section{MATERIAIS E MÉTODOS}

Num período de dois anos, foram encaminhados ao Departamento de Diagnóstico por Imagem da Universidade Federal de São Paulo/Escola Paulista de Medicina (Unifesp/EPM), 52 pacientes para tratamento percutâneo de coleções abdominais, segundo indicação da equipe clínicocirúrgica do hospital.
Destes pacientes, 16 apresentavam coleções intraperitoneais. Os sinais e sintomas mais comuns eram dor abdominal, febre, massa palpável e vômitos.

Quanto à topografia das coleções, 11 encontravam-se entre alças, três achavamse em situação subfrênica e duas, em posição sub-hepática.

A maioria das coleções (9/16) encontrava-se infectada, não havendo abscessos amebianos entre as avaliadas. Foram excluídas as coleções rotas.

Dos 16 pacientes com coleções intraperitoneais, nove $(56,3 \%)$ eram do sexo masculino e sete $(43,7 \%)$ eram do sexo feminino, com média de idade de 44 anos, variando de três meses a 66 anos.

As coleções foram diagnosticadas por métodos seccionais de imagem, por meio de US e/ou TC. A orientação dos procedimentos percutâneos foi realizada em equipamentos de ultra-sonografia modelos Tosbee ${ }^{\circledR}$ (Toshiba) e HDI Ultramark- $9^{\circledR}$ (ATL).

Os critérios para a realização do procedimento incluíram a presença de coleção definida por TC ou US sem resposta ao tratamento clínico com antibioticoterapia, via de acesso segura para a realização do procedimento e consentimento informado do paciente. A escolha do procedimento seguiu a indicação da equipe médica responsável pelo paciente, sem a participação ou interferência do médico radiologista.

$\mathrm{O}$ volume das coleções estudadas foi calculado pela fórmula da elipse (volume $=\mathrm{L} \times \mathrm{T} \times \mathrm{AP} \times 0,523)$, variando de $40 \mathrm{a}$ $1.200 \mathrm{ml}$, com média de $320 \mathrm{ml}$ e mediana de $200 \mathrm{ml}$. Das coleções avaliadas, oito $(50,0 \%)$ apresentavam volume inferior ou igual a $200 \mathrm{ml}$ e oito $(50,0 \%)$, volume superior a $200 \mathrm{ml}$.

Com relação à morfologia das coleções, seis $(37,5 \%)$ foram consideradas simples e dez $(62,5 \%)$, complexas, quando as coleções eram multiloculadas, múltiplas ou associadas a fístulas.

Os métodos percutâneos foram realizados por dois radiologistas independentes, ambos com experiência intervencionista, os quais informaram aos pacientes os riscos e benefícios do procedimento. Foram considerados aptos para o tratamento percutâneo os pacientes com número de plaquetas superior a $50.000 / \mu 1$ e tempo de protrombina inferior a 3 segundos.
A AP foi realizada em seis $(37,5 \%)$ das coleções e a DP, em dez (62,5\%). Todos os pacientes estavam em uso de antibióticos antes e após o procedimento.

Para a realização da AP foram utilizadas agulhas de punção com calibre variando de 14 a $22 \mathrm{G}$, após anestesia local com lidocaína a $1 \%$ e, em alguns casos, sedação por via oral com $10 \mathrm{mg}$ de diazepam. Iniciava-se o procedimento aspirando-se a coleção, com o objetivo de aspirar pelo menos $50 \%$ do seu volume original. Um pequeno volume do material aspirado (5 a $10 \mathrm{ml}$ ) era então encaminhado para análise laboratorial (cultura e pesquisa do microorganismo).

Na realização da DP, após anestesia local com lidocaína a $1 \%$, e em alguns casos sedação por via oral com $10 \mathrm{mg}$ de diazepam, utilizaram-se agulhas de punção com calibre de 14 a 22 G, com aspiração de 5 a $10 \mathrm{ml}$ do conteúdo da coleção e envio para análise laboratorial. Então, procedia-se à introdução do cateter de drenagem (8 a 12 F), segundo a técnica de Trocar ou de Seldinger.

O radiologista envolvido no procedimento encarregava-se, junto à equipe clínico-cirúrgica, do acompanhamento clínico-laboratorial do paciente, com controle da drenagem, lavagens diárias do dreno com 5 a $10 \mathrm{ml}$ de soro fisiológico a $0,9 \%$ e aspiração. $\mathrm{O}$ dreno era retirado assim que o débito de drenagem atingia valores inferiores a $5 \mathrm{ml}$ em 24 horas, por dois dias consecutivos, com o paciente apresentando boa evolução clínica e laboratorial e com normalização do hemograma e da temperatura. Nos casos de evolução desfavorável realizavam-se controles radiológicos com US e/ou TC.

Os resultados da evolução clínica dos pacientes submetidos aos métodos percutâneos foram classificados em:

- Sucesso: resolução completa da coleção e melhora do quadro clínico e laboratorial do paciente, sendo este acompanhado por até um ano (Figuras 1 e 2).

- Falha: ausência de preenchimento dos critérios de sucesso (Figura 3).

A análise estatística foi considerada inapropriada, pela limitada casuística. Em alguns casos, a simples observação dos dados foi suficiente para uma análise adequada desses casos. 

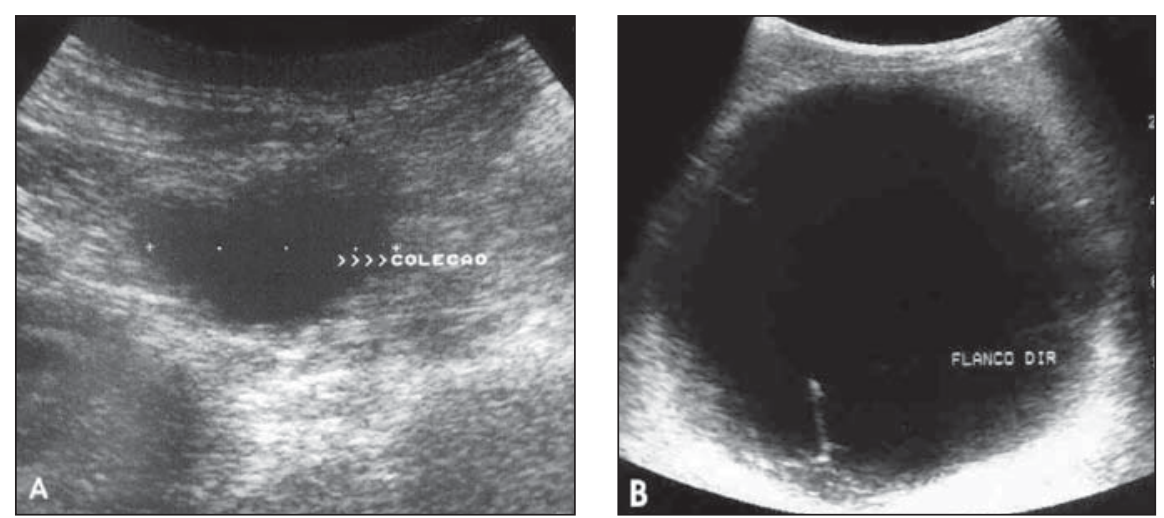

Figura 1. Coleções simples submetidas a aspiração percutânea, com sucesso.
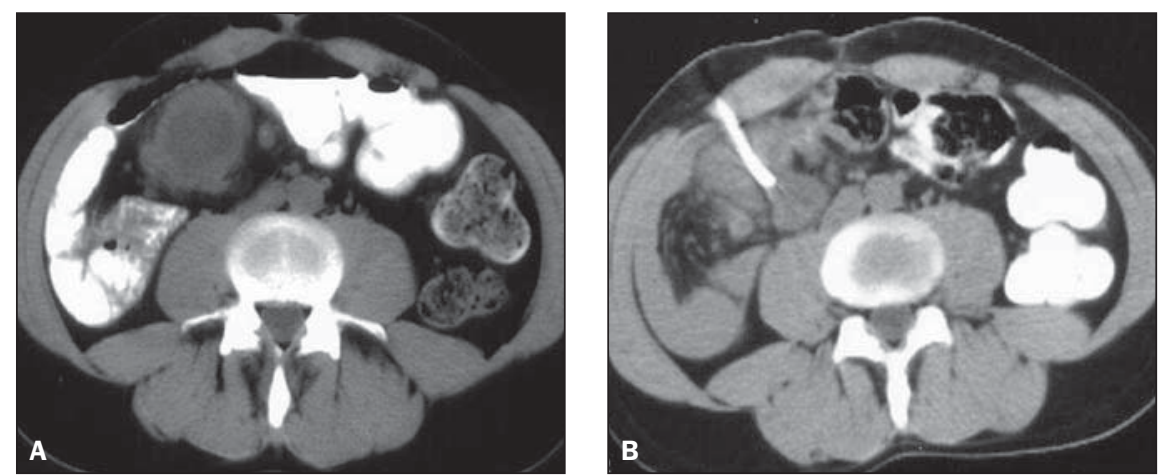

Figura 2. A: Coleção simples submetida a drenagem percutânea. B: Controle por tomografia computadorizada evidencia redução das dimensões da coleção e sucesso do método.
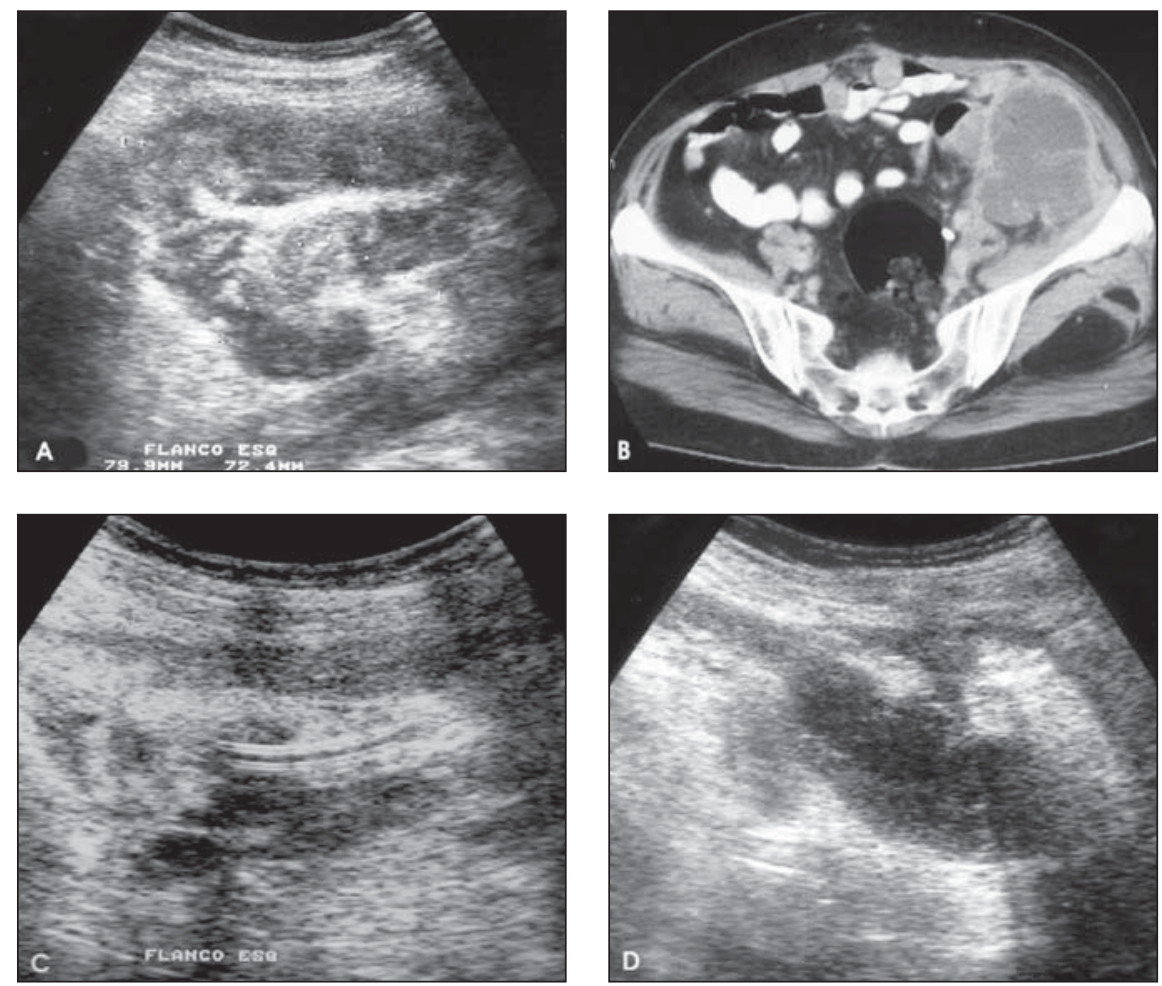

Figura 3. A,B: Coleção complexa no flanco esquerdo, submetida a drenagem percutânea. C: Drenagem da coleção, sem complicações, com manutenção do dreno. D: Recidiva da coleção, caracterizando falha do procedimento percutâneo.

O protocolo do estudo foi aprovado, sem restrições, pela Comissão de Ética Médica do Hospital São Paulo, da Unifesp/ EPM.

\section{RESULTADOS}

Analisando-se os resultados globais do tratamento das coleções intraperitoneais (Tabela 1), podemos constatar que houve sucesso em $12(75,0 \%)$ coleções e falha em quatro $(25,0 \%)$. A AP obteve sucesso em todos os casos, enquanto a DP falhou em quatro $(40,0 \%)$ coleções. Os insucessos com a DP se deveram a recidiva após seis meses, em um paciente, e a multiloculações com recidiva, nos demais. Ocorreram apenas complicações leves (bacteremia transitória e infecção cutânea) em dois pacientes submetidos a DP.

Tabela 1 Resultado do tratamento das coleções intraperitoneais, segundo o tipo de procedimento.

\begin{tabular}{|c|c|c|c|c|}
\hline \multirow{2}{*}{$\begin{array}{l}\text { Resultado } \\
\text { final }\end{array}$} & \multicolumn{4}{|c|}{ Procedimento } \\
\hline & $\begin{array}{ll} & \text { AP } \\
N & (\%)\end{array}$ & & $\begin{array}{l}\mathrm{DP} \\
(\%)\end{array}$ & $\begin{array}{c}\text { Total } \\
\text { N }(\%)\end{array}$ \\
\hline Sucesso & $6(100,0)$ & & $(60,0)$ & $12(75,0)$ \\
\hline Falha & $0(0,0)$ & 4 & $(40,0)$ & $4(25,0)$ \\
\hline Total & $6(100,0)$ & 10 & $(100,0)$ & $16(100,0)$ \\
\hline
\end{tabular}

AP, aspiração percutânea; DP, drenagem percutânea.

Segundo a mediana, as coleções foram divididas em dois grupos: aquelas com volume inferior ou igual a $200 \mathrm{ml} \mathrm{e}$ as com volume superior a $200 \mathrm{ml}$. Comparandose os resultados do tratamento percutâneo nos dois grupos, não observamos alteração no índice de sucesso com a AP, que foi sempre de $100 \%$. A DP, por outro lado, foi ligeiramente mais eficaz para as coleções de maior volume $(75,0 \%)$ do que para as de menor volume $(25,0 \%)$, apesar desta diferença não ter sido considerada significante (Tabelas 2 e 3 ).

Tabela 2 Resultado do tratamento das coleções com volume menor ou igual a $200 \mathrm{ml}$, segundo o tipo de procedimento.

\begin{tabular}{|l|l|l|l|}
\hline \multirow{2}{*}{$\begin{array}{c}\text { Resultado } \\
\text { final }\end{array}$} & \multicolumn{4}{|c|}{ Procedimento } \\
\cline { 2 - 5 } & \multicolumn{2}{|c|}{$\begin{array}{c}\text { AP } \\
(\%)\end{array}$} & \multicolumn{2}{|c|}{$\begin{array}{c}\text { DP } \\
(\%)\end{array}$} & $\begin{array}{c}\text { Total } \\
(\%)\end{array}$ \\
\hline Sucesso & $2(100,0)$ & $3(50,0)$ & $5(62,5)$ \\
Falha & $0(0,0)$ & $3(50,0)$ & $3(37,5)$ \\
\hline Total & $2(100,0)$ & $6(100,0)$ & $8(100,0)$ \\
\hline
\end{tabular}

AP, aspiração percutânea; DP, drenagem percutânea. 
Tabela 3 Resultado do tratamento das coleções com volume maior que $200 \mathrm{ml}$, segundo o tipo de procedimento.

\begin{tabular}{|l|l|l|l|}
\hline \multirow{2}{*}{$\begin{array}{l}\text { Resultado } \\
\text { final }\end{array}$} & \multicolumn{4}{|c|}{ Procedimento } \\
\cline { 2 - 5 } & \multicolumn{2}{|c|}{$\begin{array}{c}\text { AP } \\
(\%)\end{array}$} & \multicolumn{2}{|c|}{$\begin{array}{c}\text { DP } \\
(\%)\end{array}$} & $\begin{array}{c}\text { Total } \\
(\%)\end{array}$ \\
\hline Sucesso & $4(100,0)$ & $3(75,0)$ & $7(87,5)$ \\
Falha & $0(0,0)$ & $1(25,0)$ & $1(12,5)$ \\
\hline Total & $4(100,0)$ & $4(100,0)$ & $8(100,0)$ \\
\hline
\end{tabular}

AP, aspiração percutânea; DP, drenagem percutânea.

Da mesma forma, de acordo com a morfologia e aspecto das coleções, estas foram divididas nos seguintes grupos: coleções intraperitoneais simples e complexas e coleções infectadas e não infectadas.

Nas coleções submetidas a AP não observamos diferença no índice de sucesso, de $100 \%$, para coleções simples ou complexas ou para coleções não infectadas e infectadas. Com a DP, entretanto, notamos tendência a um maior índice de sucesso para coleções simples $(100,0 \%)$, quando comparadas às complexas, nas quais o sucesso ocorreu em apenas $33,3 \%$ dos casos (Tabelas 4 e 5).

Notamos ainda tendência a um menor índice de sucesso com a DP no grupo das coleções infectadas, nas quais falhas ocorreram em $57,1 \%$ das coleções, não havendo casos de insucesso nas não infectadas (Tabelas 6 e 7).

\section{DISCUSSÃO}

As coleções intraperitoneais são afecções que requerem tratamento rápido e eficaz, devido aos altos índices de mortalidade se não são adequadamente diagnosticadas e tratadas ${ }^{(1,3,12)}$. Com o surgimento de novos métodos de imagem, proporcionando informações adicionais, diagnósticos mais precoces e acurados podem ser estabelecidos, permitindo intervenção ime$\operatorname{diata}^{(3,12,14)}$.

Com o advento de modernos métodos de imagem, surgiram novas técnicas para tratamento das coleções intraperitoneais pela via percutânea, evitando o trauma cirúrgico e anestésico e evoluindo com menores índices de morbidade, complicações e mortalidade em relação às técnicas cirúrgicas $^{(4,11-13)}$. A cirurgia continua a desempenhar papel de grande relevância, devendo ser considerada a alternativa terapêuti-
Tabela 4 Resultado do tratamento das coleções simples, segundo o tipo de procedimento.

\begin{tabular}{|l|l|l|l|}
\hline \multirow{2}{*}{$\begin{array}{l}\text { Resultado } \\
\text { final }\end{array}$} & \multicolumn{4}{|c|}{ Procedimento } \\
\cline { 2 - 5 } & \multicolumn{2}{|c|}{$\begin{array}{c}\text { AP } \\
(\%)\end{array}$} & \multicolumn{2}{|c|}{$\begin{array}{c}\text { DP } \\
(\%)\end{array}$} & \multicolumn{1}{c|}{$\begin{array}{c}\text { Total } \\
(\%)\end{array}$} \\
\hline Sucesso & $2(100,0)$ & $4(100,0)$ & $6(100,0)$ \\
Falha & $0(0,0)$ & $0(0,0)$ & $0(0,0)$ \\
\hline Total & $2(100,0)$ & $4(100,0)$ & $6(100,0)$ \\
\hline
\end{tabular}

AP, aspiração percutânea; DP, drenagem percutânea.

Tabela 6 Resultado do tratamento das coleções não infectadas, segundo o tipo de procedimento.

\begin{tabular}{|l|l|l|l|}
\hline \multirow{2}{*}{$\begin{array}{c}\text { Resultado } \\
\text { final }\end{array}$} & \multicolumn{4}{|c|}{ Procedimento } \\
\cline { 2 - 5 } & \multicolumn{2}{|c|}{$\begin{array}{c}\text { AP } \\
(\%)\end{array}$} & \multicolumn{2}{c|}{$\begin{array}{c}\text { DP } \\
(\%)\end{array}$} & \multicolumn{1}{|c|}{$\begin{array}{c}\text { Total } \\
(\%)\end{array}$} \\
\hline Sucesso & $4(100,0)$ & $3(100,0)$ & $7(100,0)$ \\
Falha & $0(0,0)$ & $0(0,0)$ & $0(0,0)$ \\
\hline Total & $4(100,0)$ & $3(100,0)$ & $7(100,0)$ \\
\hline
\end{tabular}

AP, aspiração percutânea; DP, drenagem percutânea.

ca quando não há acesso percutâneo seguro, para coleções multiloculadas ou múltiplas que exijam debridamento manual e nos casos de complicações ou falhas dos procedimentos percutâneos ${ }^{(1,2,4,4)}$.

Apesar de vários estudos compararem os resultados obtidos com o tratamento cirúrgico e com os métodos percutâneos guiados por técnicas de imagem na abordagem das coleções intraperitoneais ${ }^{\mathbf{4}, \mathbf{6 , 1 2} \text {, }}$ ${ }^{13,16)}$, raros são aqueles que comparam os resultados obtidos com a AP e a $\mathrm{DP}^{(9)}$. A indicação do procedimento percutâneo (drenagem ou aspiração) deve ser sempre uma escolha multidisciplinar, baseando-se principalmente na localização, possibilidade de via de acesso, volume e natureza da lesão ${ }^{(4)}$. A AP apresenta vantagens por ser um procedimento mais simples, cômodo, barato e rápido, causando menos desconforto ao paciente e exigindo menor controle médico e de enfermagem em relação à $\mathrm{DP}^{(9,22,23)}$. Além disso, com a AP, múltiplas coleções ou cavidades podem ser aspiradas simultaneamente ${ }^{(9,24)}$.

A DP, embora apresente algumas desvantagens em relação à $\mathrm{AP}$, como custo mais elevado, maior risco de infecção, lesão de estruturas e formação de hematomas ${ }^{(22,24)}$, permite melhor e mais prolongado manuseio das coleções, com drenagem contínua, controle do esvaziamento e pequena possibilidade de reacúmulo ou dre-

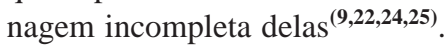

Tabela 5 Resultado do tratamento das coleções complexas, segundo o tipo de procedimento.

\begin{tabular}{|l|l|l|c|}
\hline \multirow{2}{*}{$\begin{array}{c}\text { Resultado } \\
\text { final }\end{array}$} & \multicolumn{4}{|c|}{ Procedimento } \\
\cline { 2 - 5 } & $\mathrm{N}$ & $\begin{array}{c}\text { AP } \\
(\%)\end{array}$ & \multicolumn{2}{|c|}{$\begin{array}{c}\text { DP } \\
(\%)\end{array}$} & \multicolumn{2}{c|}{$\begin{array}{c}\text { Total } \\
(\%)\end{array}$} \\
\hline Sucesso & $4(100,0)$ & $2(33,3)$ & $6(60,0)$ \\
Falha & $0(0,0)$ & $4(66,7)$ & $4(40,0)$ \\
\hline Total & $4(100,0)$ & $6(100,0)$ & $10(100,0)$ \\
\hline
\end{tabular}

AP, aspiração percutânea; DP, drenagem percutânea.

Tabela 7 Resultado do tratamento das coleções infectadas, segundo o tipo de procedimento.

\begin{tabular}{|l|l|l|l|}
\hline \multirow{2}{*}{$\begin{array}{l}\text { Resultado } \\
\text { final }\end{array}$} & \multicolumn{4}{|c|}{ Procedimento } \\
\cline { 2 - 5 } & \multicolumn{2}{|c|}{$\begin{array}{c}\text { AP } \\
(\%)\end{array}$} & \multicolumn{2}{|c|}{$\begin{array}{c}\text { DP } \\
(\%)\end{array}$} & $\begin{array}{c}\text { Total } \\
(\%)\end{array}$ \\
\hline Sucesso & $2(100,0)$ & $3(42,9)$ & $5(55,5)$ \\
Falha & $0(0,0)$ & $4(57,1)$ & $4(44,5)$ \\
\hline Total & $2(100,0)$ & $7(100,0)$ & $9(100,0)$ \\
\hline
\end{tabular}

AP, aspiração percutânea; DP, drenagem percutânea.

Apesar das vantagens da AP em relação à DP, o método não ganhou popularidade. A grande maioria dos médicos aceita o tratamento percutâneo, mas insiste na manutenção de cateteres de grande calibre no tratamento das coleções, o que não é possível com a aspiração percutânea, apenas com a drenagem ${ }^{(8,13)}$.

Gronvall et al. ${ }^{(9)}$ trataram 50 coleções abdominais pela via percutânea, com $82 \%$ de sucesso, não se observando diferença significativa entre a AP e a DP. No nosso estudo, apesar da menor casuística, observamos maior índice de sucesso com a AP (100\%) do que com a DP (60\%) para tratamento percutâneo de 16 coleções intraperitoneais.

O menor índice de sucesso da drenagem em relação à aspiração percutânea no nosso estudo pode estar relacionado ao fato de coleções mais complexas, como as múltiplas, associadas a fístulas ou multisseptadas, terem sido selecionadas para drenagem, já que a escolha do método baseavase na indicação da equipe clínico-cirúrgica do hospital.

Não observamos diferença significante no índice de sucesso com a variação do volume da coleção, tanto para a AP quanto para a DP.

No que se refere à morfologia e natureza da coleção, entretanto, notou-se tendência a um maior índice de falhas da DP para coleções complexas e infectadas. Com 
este procedimento, o índice de sucesso foi significativamente maior nas coleções simples $(100 \%)$ e nas não infectadas $(100 \%)$ do que nas complexas $(33,3 \%)$ ou infectadas $(42,9 \%)$. Em contraste, nas coleções submetidas à AP, não houve casos de esvaziamento incompleto ou reacúmulo da coleção, com sucesso elevado (100\%) em todos os subgrupos.

Não encontramos relatos na literatura comparando a AP e a DP conforme o subgrupo das coleções intraperitoneais, em relação a volume, complexidade e natureza dessas coleções.

É interessante ressaltar que os resultados para coleções intraperitoneais são bem diferentes dos obtidos com os mesmos métodos percutâneos para coleções hepáticas, segundo estudo que realizamos ${ }^{(26)}$. Neste, constatamos um maior índice de sucesso global da DP, principalmente para coleções hepáticas mais volumosas e complexas. É por esta razão que acreditamos que as coleções intraperitoneais mais complexas tenham sido selecionadas para drenagem pela equipe clínico-cirúrgica.

Apesar de raras, algumas complicações são descritas, tanto com a DP quanto com a AP, mas a maioria não requer intervenção cirúrgica ${ }^{(\mathbf{1 0 , 1 5 , 2 7 )}}$. No nosso estudo, observamos apenas duas complicações, menores e transitórias, com a DP, sugerindo que ambos os métodos sejam opções seguras na terapia das coleções intraperitoneais. É importante salientar, mais uma vez, que a opção pelo tratamento percutâneo dependerá do local, acesso, volume e natureza da coleção, e que algumas coleções não são acessíveis a esse tratamento, pelo elevado risco de lesão de estruturas vitais.

Maior experiência será necessária para validar nossas impressões iniciais, pela reduzida casuística do estudo, que impossibilita inclusive a aplicação de testes esta- tísticos mais acurados. Entretanto, na maioria dos casos, a diferença entre os métodos pode ser sensivelmente observada, permitindo uma comparação confiável.

\section{CONCLUSÃO}

A AP e a DP são métodos eficazes e seguros para tratamento das coleções intraperitoneais. No nosso estudo, a AP foi mais eficaz que a DP, principalmente nas coleções de menor volume.

\section{REFERÊNCIAS}

1. Adil A, Ousehal A, Essodegui F, Abdelouafi A, Kadiri R. Traitement percutané des collections abdominales. A propos de 135 cas. J Radiol 1995;76: 129-34.

2. Gerzof SG, Robbins AH, Johnson WC, Birkett DH, Nabseth DC. Percutaneous catheter drainage of abdominal abscesses: a five-year experience. NEngl J Med 1981;305:653-7.

3. Montgomery RS, Wilson SE. Intraabdominal abscesses: image-guided diagnosis and therapy. Clin Infect Dis 1996;23:28-36.

4. Ayerza HA. Abcès intra-abdominaux. A propos de 530 cas. Chirurgie 1994;120:551-4.

5. Altemeier WA, Culbertson WR, Fullen WD, Shook CD. Intra-abdominal abscesses. Am J Surg 1973; 125:70-9.

6. Levison MA. Percutaneous versus open operative drainage of intra-abdominal abscesses. Infect Dis Clin North Am 1992;6:525-44.

7. Gandon Y, Mueller PR, Ferrucci JT. Abscess and intra-abdominal fluid collections. Diagnosis and percutaneous drainage. J Radiol 1989;70:235-47.

8. Sones PJ. Percutaneous drainage of abdominal abscesses. AJR 1984;142:35-9.

9. Gronvall S, Gammelgaard J, Haubek A, Holm HH. Drainage of abdominal abscesses guided by sonography. AJR 1982;138:527-9.

10. Lambiase RE, Deyoe L, Cronan JJ, Dorfman GS. Percutaneous drainage of 335 consecutive abscesses: results of primary drainage with 1-year followup. Radiology 1992;184:167-79.

11. Kopecky KK, Wass JL, Cory DA, Baker MK, Jackson VP, Goulet RJ Jr. Percutaneous drainage of intra-abdominal and retroperitoneal abscesses. Indiana Med 1987;80:1168-71.

12. Vadala G, Caragliano V, Castorina R, Caragliano L, Caragliano P. An update in the treatment of intraabdominal abscesses. Minerva Chir 1999;54:24550 .
13. vanSonnenberg E, Ferrucci JT Jr, Mueller PR, Wittenberg J, Simeone JF. Percutaneous drainage of abscesses and fluid collections: technique, results, and applications. Radiology 1982;142:1-10.

14. Dondelinger RF. Advances in abdominal interventional radiology. Lancet 1999;353 Suppl 1:S15-8.

15. Lurie K, Plzak L, Deveney CW. Intra-abdominal abscess in the 1980s. Surg Clin North Am 1987; 67:621-32

16. Sahai A, Belair M, Gianfelice D, Cote S, Gratton J, Lahaie R. Percutaneous drainage of intra-abdominal abscesses in Crohn's disease: short and longterm outcome. Am J Gastroenterol 1997;92:2758.

17. Holm HH, Pedersen JF, Kristensen JK, Rasmussen SN, Hancke S, Jensen F. Ultrasonically guided percutaneous puncture. Radiol Clin North Am 1975 13:493-503.

18. Kuligowska E, Connors SK, Shapiro JH. Liver abscess: sonography in diagnosis and treatment. AJR 1982;138:253-7.

19. Maher JA Jr, Reynolds TB, Yellin AE. Successful medical treatment of pyogenic liver abscess. Gastroenterology 1979;77(4 Pt 1):618-22.

20. Smith EH, Bartrum RJ Jr. Ultrasonically guided percutaneous aspiration of abscesses. Am J Roentgenol Radium Ther Nucl Med 1974;122:308-12.

21. Elyaderani MK, Skolnick ML, Weinstein BJ. Ultrasonic detection and aspiration confirmation of intra-abdominal collection of fluid. Surg Gynecol Obstet 1979;149:529-33.

22. Baek SY, Lee MG, Cho KS, Lee SC, Sung KB, Auh YH. Therapeutic percutaneous aspiration of hepatic abscesses: effectiveness in 25 patients. AJR 1993; 160:799-802.

23. Berger LA, Osborne DR. Treatment of pyogenic liver abscesses by percutaneous needle aspiration. Lancet 1982;1(8264):132-4.

24. Rajak CL, Gupta S, Jain S, Chawla Y, Gulati M, Suri S. Percutaneous treatment of liver abscesses: needle aspiration versus catheter drainage. AJR 1998;170: 1035-9.

25. Bernardino ME, Berkman WA, Plemmons M, Sones PJ Jr, Price RB, Casarella WJ. Percutaneous drainage of multiseptated hepatic abscess. J Comput Assist Tomogr 1984;8:38-41.

26. Mello GGN, Meirelles GSP, Oliveira JMA, Ajzen S, D'Ippolito G, Szejnfeld J. Estudo comparativo dos procedimentos percutâneos orientados por métodos de imagem no tratamento das coleções hepáticas. Radiol Bras 2001;34:83-8.

27. Piette JC, Saba J, Perronne C, Le Charpentier Y. Anatomo-clinical conference. Hopital BichatClaude Bernard. Case No. 2- 1993. Recurrent subcutaneous and peritoneal abscesses. Ann Med Interne 1993;144:251-6. 